\title{
'Down-triage' for children with abnormal vital signs: evaluation of a new triage practice at a paediatric emergency department in Japan
}

\author{
Takuto Takahashi, ${ }^{1}$ Nobuaki Inoue, ${ }^{2}$ Naoki Shimizu, ${ }^{3}$ Toshiro Terakawa, ${ }^{1}$ \\ Ran D Goldman ${ }^{4}$
}

${ }^{1}$ Division of General Pediatrics, Department of Pediatrics, Tokyo Metropolitan Children's Medical Center, Fuchu, Tokyo, Japan

${ }^{2}$ Division of Pediatric Emergency Medicine, Department of Pediatric Emergency and Critical Care Medicine, Fuchu, Tokyo, Japan ${ }^{3}$ Division of Pediatric Critical Care Medicine, Department of Pediatric Emergency and Critical Care Medicine, Fuchu,

Tokyo, Japan

${ }^{4}$ Pediatric Research in Emergency Therapeutics Program (PRETX.org), Division of Emergency Medicine, Department of Pediatrics, University of British Columbia, Child and Family Research Institute, Vancouver, British Columbia, Canada

\section{Correspondence to} Dr Takuto Takahashi, 2-8-29 Musashidai, Fuchu, Tokyo 183-8561, Japan; takutotakahashi0@gmail.com

Received 24 April 2015 Revised 6 March 2016 Accepted 13 March 2016 Published Online First 4 April 2016

\section{ABSTRACT}

Objective Assessment of abnormal vital signs in triage is a challenge in the paediatric emergency department (PED), since vital signs may reflect anxiety, fever or pain rather than the clinical deterioration of the child. We aimed to evaluate the efficacy of subjective 'down-triage' (change of the initially determined acuity levels) of Japanese Triage and Acuity Scale (JTAS).

Methods This is a retrospective cohort study of patients in PED up to 15 years of age at a tertiary paediatric medical centre in Japan during a 1-year period. At the end of every JTAS triage process, PED nurses were allowed to 'down-triage' acuity levels of well-appearing patients with abnormal HR or RR, which were presumably attributable to fever, crying or being upset. We compared predictive performance of the triage system before and after 'down-triage' using admission rate as the primary outcome.

Results Among 37961 PED visits during the study period, we analysed 37219 records. A total of 17089 patients $(45.9 \%)$ were 'down-triaged' after their initial triage allocation upon arrival. Admission rates after 'down-triage' $(83 \%, 33 \%, 7 \%, 1 \%$ and 3\% for levels $1-5$, respectively), compared with those of unmodified initial level $(16 \%, 11 \%, 6 \%, 2 \%$ and $6 \%$ for levels $1-5$, respectively), had a better apparent relevance with the anticipated admission rates of Canadian Triage and Acuity Scale.

Conclusions Modification of JTAS through 'downtriage' by experienced staff improves prediction of disposition in a PED. Further research is needed to determine an objective protocol for 'down-triage' to ensure safe practice in a PED.

\section{INTRODUCTION}

Emergency department (ED) triage systems prioritise patients based on their urgency through brief, initial evaluation including vital signs at presentation to the hospital. A principal challenge in paediatric triage is the assessment of abnormal vital signs. First, paediatric reference ranges of normal $\mathrm{HR}$ and RR are based on patient's age. Furthermore, stress and anxiety, experienced by many young children, may result in high HR and RR and may be perceived abnormal. Fever, the most common complaint of children coming to a paediatric ED (PED) may also result in increased HR and RR. ${ }^{12}$

Designation of higher acuity levels solely based on abnormal vital signs may result in inappropriately triaging to a higher acuity, delaying care to

\section{Key messages}

What is already known on this subject? The Canadian Triage and Acuity Scale is a well-established triage system, which incorporate presenting vital signs in prioritisation of patients in paediatric emergency departments (PED). Assessment of abnormal vital signs is difficult in PED, since vital signs may reflect anxiety, fever or pain rather than the clinical deterioration of the child. Previous studies reported that triage of paediatric patients based on abnormal vital signs may inappropriately assign higher acuity levels.

\section{What might this study add?}

In this prospective study in a paediatric emergency centre in Japan, triage nurses down-triaged $46 \%$ of all patients. The admission rates of down-triaged patients were apparently more consistent with anticipated admission rates of the triage scale, compared with initially assigned triage level.

other, more urgent, patients. A previous study from Taiwan reported over-triaging of children with nonurgent complaints due to abnormal vital signs. They postulated that over-triaging otherwise wellappearing infants and toddlers with abnormal vital signs caused a relatively low admission rate in their highest acuity level group. ${ }^{3}$ Those developing the Canadian Triage and Acuity Scale (CTAS) guidelines addressed this issue in 2008, suggesting that triaging lower acuity based on measured vital signs should be done with caution (eg, tachycardia in an upset child). ${ }^{4}$

To accommodate prioritisation of patients in face of increased demand and limited staff and space, our PED in Tokyo, Japan, implemented a modified Japanese Triage and Acuity Scale (mod-JTAS) protocol with an option to 'down-triage' (reduce level of acuity) following the initial JTAS triage process. 'Down-triage' was reserved for well-appearing children with obvious reasons for abnormal vital signs at the time of initial triage. To date, only one Canadian study documented 'down-triage' reporting that wellappearing febrile children 3-36 months old could safely be 'down-triaged' from PED CTAS level 3-4. The objective of this study was to compare the predictive performance of triage systems before and after the subjective 'down-triage', 


\section{MATERIALS AND METHODS}

The CTAS was first implemented in 1999 in Canada and most recently revised in $2014 .{ }^{6}$ CTAS assigns acuity level of $1-5$ (1 being the most urgent) using subjective and objective evaluation of chief complaint in conjunction with physiologic and historical modifiers. The CTAS National Working Group and the assembly of emergency medicine academic organizations in Japan collaborated to officially publish CTAS in Japanese in 2010 and modified to JTAS in 2012, with the addition of several presenting complaints common in Japan. The implementation of JTAS in Japan has previously reported in adult EDs. ${ }^{7}$ CTAS recently introduced entirely new paediatric reference ranges for HR and RR following the first published systematic review. ${ }^{6} 8$

This was a retrospective cohort study using an administrative electronic triage database from one tertiary paediatric medical centre in Tokyo, Japan. The PED primarily provides care to children up to 15 years of age with an annual census of approximately 38000 visits including 3200 ambulance transfers. As an educational centre, paediatric residents and paediatric emergency medicine fellows are supervised by attending physicians. We included all records of children up to 15 years of age visiting our PED over a 1-year period (1 January 2013 to 31 December 2013). We excluded children who were not triaged, those with no record of triage level or if the visit was made by parents without accompanying children (for advice purposes). We collected demographic information (age and gender) and PED visit information (day and time of visit), mode of arrival (walk-in or ambulance), triage level based on JTAS, modified JTAS (mod-JTAS) triage level after 'down-triage', precategorised indication for 'down-triage', disposition and vital signs (HR, RR and saturation $\left.\left(\mathrm{SpO}_{2}\right)\right)$ in triage.

During the study period, patients were triaged by a registered nurse using computerised version of JTAS, indicating an initial triage level based on presenting complaints, physiological and historical modifiers. All triage nurses had more than 4 years of experience, had at least 4 months of training and were certified by one of the triage nurse educators in the PED. We used axillary electronic thermometers to measure temperature, pulse oximeters for HR and RR was counted by auscultation along with visual confirmation of chest movement. We used the paediatric reference ranges for HR and RR recommended in the 2013 revision of the CTAS. ${ }^{6} 8$

Nurses were allowed to 'down-triage' acuity level at the end of the regular triage process of JTAS in every patient. The candidates for 'down-triage' were patients with initial abnormal vital signs (HR or RR) that nurses perceived well and that their vital signs were abnormal due to fever, crying or being anxious. Patients were seen by physicians based on the final triage levels assigned after 'down-triage' assessment, but the initial levels were also available to physicians in triage notes.

The primary outcome was overall admissions per JTAS and mod-JTAS levels. Secondary outcome measures included ICU admission per JTAS and mod-JTAS levels, rates of transition from JTAS to mod-JTAS levels and comparison of overall admission rates per JTAS and mod-JTAS levels with those of a previous study on Canadian PEDs and the anticipated rates defined by CTAS. Other variables included patients left without being seen (LWBS), age, gender, the time of arrival (0:00-8:00, 8:00$16: 00$ or $16: 00-24: 00)$, final disposition and arrival by ambulance.

\section{Data analysis}

We used Microsoft Excel 97-2003 (Redmond, Washington, USA). Statistical analysis was carried out using SPSS software (V.22.0; SPSS, Chicago, Illinois, USA). We used $\chi^{2}$ test for comparison of categorical variables, and Student's $t$ test or MannWhitney $\mathrm{U}$ test for continuous variables. A $\mathrm{p}$ value $<0.05$ was considered to be statistically significant. This study was approved by the ethical committee of Tokyo Metropolitan Children's Medical Center.

\section{RESULTS}

We reviewed a total of 37961 records of PED visits including $37450(98.7 \%)$ for patients up to 15 years of age. We excluded $231(0.6 \%)$ patients because of lack of documented triage levels (128), patients not triaged (67) and visits made by parents without accompanying children (36) ( figure 1).

Baseline characteristics of participants are presented in table 1. A total of $2478(6.7 \%)$ were admitted to the general wards, $210(0.6 \%)$ were admitted to the ICU and $62(0.2 \%)$ were LWBS.
Figure 1 Study participants. PED, paediatric emergency department, JTAS, Japanese Triage and Acuity Scale, mod-JTAS, modified JTAS.

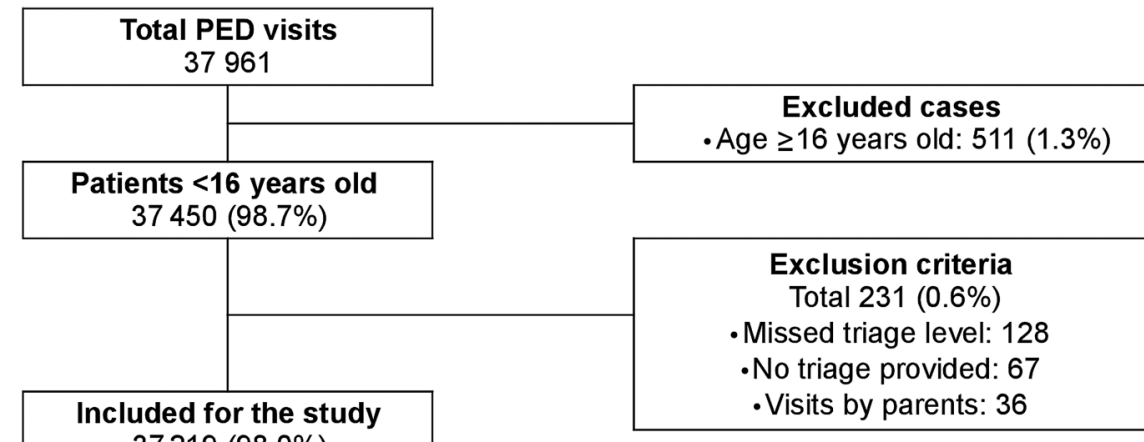

$37219(98.0 \%)$

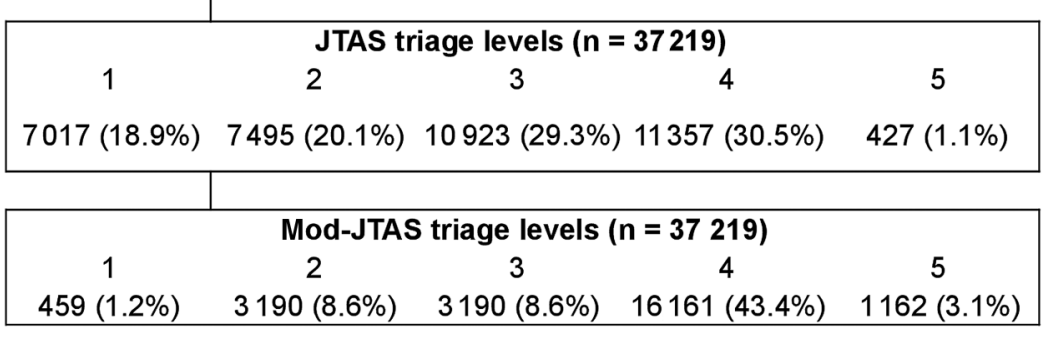


Table 1 Baseline characteristics of the participants

\begin{tabular}{|c|c|c|c|c|}
\hline \multirow[b]{2}{*}{ Characteristics } & \multirow[b]{2}{*}{$\begin{array}{l}\text { Participants } \\
\text { n=37 } 219\end{array}$} & \multicolumn{2}{|l|}{ Down-triage } & \multirow[b]{2}{*}{$p$ Value* } \\
\hline & & $\begin{array}{l}(+) \\
n=17089\end{array}$ & $\begin{array}{l}(-) \\
n=20130\end{array}$ & \\
\hline Male gender, $\mathrm{n}(\%)$ & $21149(56.8)$ & $9286(54.3)$ & $11863(58.9)$ & $<0.001$ \\
\hline Age in months, mean (SD) & $54.6(46.3)$ & $52(43.2)$ & $56.9(48.7)$ & \\
\hline Age in years, mean (SD) & $4.1(3.9)$ & $3.9(3.6)$ & $4.3(4.1)$ & \\
\hline \multicolumn{5}{|l|}{ Age group, $\mathrm{n}(\%)$} \\
\hline $0-28$ days old & $296(0.8)$ & $82(0.5)$ & $214(1.1)$ & $<0.001$ \\
\hline $1-12$ months old & $5169(13.9)$ & $1836(10.7)$ & $3333(16.6)$ & \\
\hline $1-6$ years old & $21278(57.2)$ & $10925(63.9)$ & $10353(51.4)$ & \\
\hline $6-12$ years old & $7836(21.1)$ & $3283(19.2)$ & $4553(22.6)$ & \\
\hline $12-16$ years old & $2640(7.1)$ & $963(5.6)$ & $1677(8.3)$ & \\
\hline Arrival by ambulance, $\mathrm{n}(\%)$ & $3247(8.7)$ & $1253(7.3)$ & $1994(9.9)$ & $<0.001$ \\
\hline \multicolumn{5}{|l|}{ Shift of arrival, n (\%) } \\
\hline Day (8:00-16:00) & $14950(40.2)$ & $6505(38.1)$ & $8445(42.0)$ & $<0.001$ \\
\hline Evening (16:00-midnight) & $17779(47.8)$ & $8291(48.5)$ & $9488(47.1)$ & \\
\hline Night (midnight-8:00) & $4490(12.1)$ & $2293(13.4)$ & $2197(10.9)$ & \\
\hline \multicolumn{5}{|l|}{ Final disposition, n (\%) } \\
\hline Discharge & $34350(92.3)$ & $15971(93.5)$ & $18379(91.3)$ & $<0.001$ \\
\hline Admission to general wards & $2478(6.7)$ & $1033(6.0)$ & $1445(7.2)$ & \\
\hline ICU & $210(0.6)$ & $25(0.1)$ & $185(0.9)$ & \\
\hline Transfer & $90(0.2)$ & $37(0.2)$ & $53(0.3)$ & \\
\hline LWBS & $62(0.2)$ & $19(0.1)$ & $43(0.2)$ & \\
\hline Admissions to psychiatry wards & $29(0.1)$ & $4(0.0)$ & $25(0.1)$ & \\
\hline
\end{tabular}

${ }^{*}$ Comparison between down-triage (+) versus down-triage (-).

JTAS, Japanese Triage and Acuity Scale; LWBS, left without being seen; mod-JTAS, modified JTAS.

Transition from JTAS levels to mod-JTAS levels is shown in table 2. Overall, 17089 patients (45.9\%) were 'down-triaged', 19465 patients $(52.3 \%)$ had an unchanged triage level and 665 $(1.8 \%)$ were 'up-triaged'. The higher the acuity of the patient at presentation, the higher the frequency of 'down-triage' recorded (93.7\%, 77.2\%, 38.1\% and 5.0\% for levels 1-4, respectively). Common reasons for 'down-triaging' were (1) nurses considered excessive tachycardia or tachypnoea but the child looked well (84.5\%: 14 438/17 089), (2) inappropriate bradycardia or low RR (6.1\%: 1035/17 089), (3) inappropriate low saturation (1.4\%: 243/17 089), (4) combination of (1)-(3) (5.3\%: 911/ $17089)$ and (4) other reasons (2.7\%: 462/17 089).

We compared the overall and ICU admission rates per JTAS and mod-JTAS triage levels (table 3 ). There were statistically significant differences in both overall and ICU admission rates between JTAS and mod-JTAS systems.

Mod-JTAS acuity levels, rather than JTAS levels, had an apparent better relevance with overall admission rate (figure 2).
We conducted detailed analysis on the admission rates between the 'down-triaged' versus 'non-down-triaged' at each mod-JTAS level $(35.6 \%$ vs $30.8 \%, 6.2 \%$ vs $8.5 \%$ and $0.8 \%$ vs $1.4 \%$, for levels 2,3 , and 4 and 5 , respectively).

\section{DISCUSSION}

We documented significant differences in admission rates per triage levels before and after 'down-triage' procedure. Acuity levels after 'down-triage' had a better apparent relevance with anticipated admission rates by CTAS. Down-triage practice aims to better assign an acuity level based on the true condition of the child, rather than based on variability in vital signs. 'Down-triage' process allows better prediction of disposition of children arriving to our PED. To our knowledge, this is the first report on subjective 'down-triaging' practices based on abnormal vital signs in a PED.

Considering the prioritising nature of a triage system, the impact of 'down-triage' should be assessed based on the

Table 2 Distribution of JTAS triage levels and mod-JTAS triage levels, $\mathrm{n}(\%$ of the total number in each JTAS level)

\begin{tabular}{|c|c|c|c|c|c|c|}
\hline \multirow[b]{2}{*}{ JTAS triage level } & \multicolumn{6}{|c|}{ Mod-JTAS triage level } \\
\hline & 1 & 2 & 3 & 4 & 5 & Total \\
\hline 1 & $440(6.3)$ & 1371 (19.5) & 4819 (68.7) & $377(5.4)$ & $10(0.1)$ & $7017(100$ \\
\hline 2 & $17(0.2)$ & $1689(22.5)$ & $4237(56.5)$ & $1528(20.4)$ & $24(0.3)$ & $7495(100$ \\
\hline 3 & $1(-)$ & $86(0.8)$ & $6678(61.1)$ & $4019(36.8)$ & 139 (1.3) & $10923(100$ \\
\hline 4 & $1(-)$ & $44(0.4)$ & $513(4.5)$ & $10234(90.1)$ & $565(5.0)$ & $11357(100$ \\
\hline 5 & $0(-)$ & $0(-)$ & $0(-)$ & $3(0.7)$ & 424 (99.3) & $427(100$ \\
\hline Total & 459 (1.2) & 3190 (8.6) & $16247(43.7)$ & 16161 (43.4) & $1162(3.1)$ & $37219(100)$ \\
\hline
\end{tabular}

JTAS, Japanese Triage and Acuity Scale; mod-JTAS, modified JTAS. 
Table 3 Overall and ICU admission rates per JTAS and mod-JTAS triage levels, \% of the total numbers in each level $(95 \% \mathrm{CI})$

\begin{tabular}{|c|c|c|c|c|c|c|c|c|c|c|}
\hline \multirow{2}{*}{$\begin{array}{l}\text { Triage } \\
\text { level }\end{array}$} & \multicolumn{4}{|c|}{ Overall admission rate } & \multirow[b]{2}{*}{$\mathrm{p}$ Value } & \multicolumn{4}{|c|}{ ICU admission rate } & \multirow[b]{2}{*}{$p$ Value } \\
\hline & JTAS & & Mod-JT & & & JTAS & & Mod-JT & & \\
\hline 1 & $16.2 \%$ & (15.4 to 17.1$)$ & $82.8 \%$ & (79.1 to 86.0 ) & $<0.001$ & $1.7 \%$ & (1.4 to 2.0 ) & $22.7 \%$ & (19.1 to 26.7 ) & $<0.001$ \\
\hline 2 & $10.9 \%$ & (10.2 to 11.7$)$ & $32.9 \%$ & (31.3 to 34.5 ) & & $0.8 \%$ & (0.6 to 1.0$)$ & $2.3 \%$ & (1.8 to 2.8 ) & \\
\hline 3 & $5.5 \%$ & (5.1 to 5.9 ) & $7.2 \%$ & (68.1 to 76.1 ) & & $0.2 \%$ & (0.2 to 0.4 ) & $0.2 \%$ & (0.1 to 0.3 ) & \\
\hline 4 & $2.0 \%$ & (1.8 to 2.3 ) & $1.1 \%$ & (0.9 to 1.3 ) & & $0.1 \%$ & (0.0 to 0.1 ) & $0.0 \%$ & $(0.0$ to 0.0$)$ & \\
\hline 5 & $6.1 \%$ & (4.2 to 8.8$)$ & $2.8 \%$ & (2.0 to 4.0$)$ & & $0.0 \%$ & $(0.0$ to 0.0$)$ & $0.0 \%$ & (0.0 to 0.0$)$ & \\
\hline
\end{tabular}

JTAS, Japanese Triage and Acuity Scale; mod-JTAS, modified JTAS.

predictive performance of a 5-level scale system, rather than at each level. Our overall admission rate per mod-JTAS levels $(83 \%, 33 \%, 7 \%, 1 \%$ and $3 \%$ for levels $1-5$, respectively) is similar to the anticipated admission rates by CTAS (70-90\%, $40-70 \%, 20-40 \%, 10-20 \%$ and $0-10 \%$ for levels $1-5$, respectively). ${ }^{9}$ The pooled admission rates from 12 Canadian PEDs were also comparable with our data $(61 \%, 30 \%, 10 \%, 2 \%$ and $0.9 \%$ for levels $1-5$, respectively). ${ }^{10}$

According to CTAS guidelines, assessment of vital signs (HR and RR) should be done at rest. ${ }^{9}$ However, patients in a PED are rarely comfortable due to physical and psychological stresses. Moreover, increased HR or RR in febrile patients can be a normal physiological reaction, rather than a sign of haemodynamic instability or respiratory distress. ${ }^{18}$ One study postulated that stress-induced tachycardia or tachypnoea may result in assigning triage level 1 to non-urgent patients. ${ }^{3}$ For this reason, Thompson et $a l^{2}$ derived temperature and age appropriate HR centiles for children with acute infections in an ambulatory setting. Yet, the same team reported that those

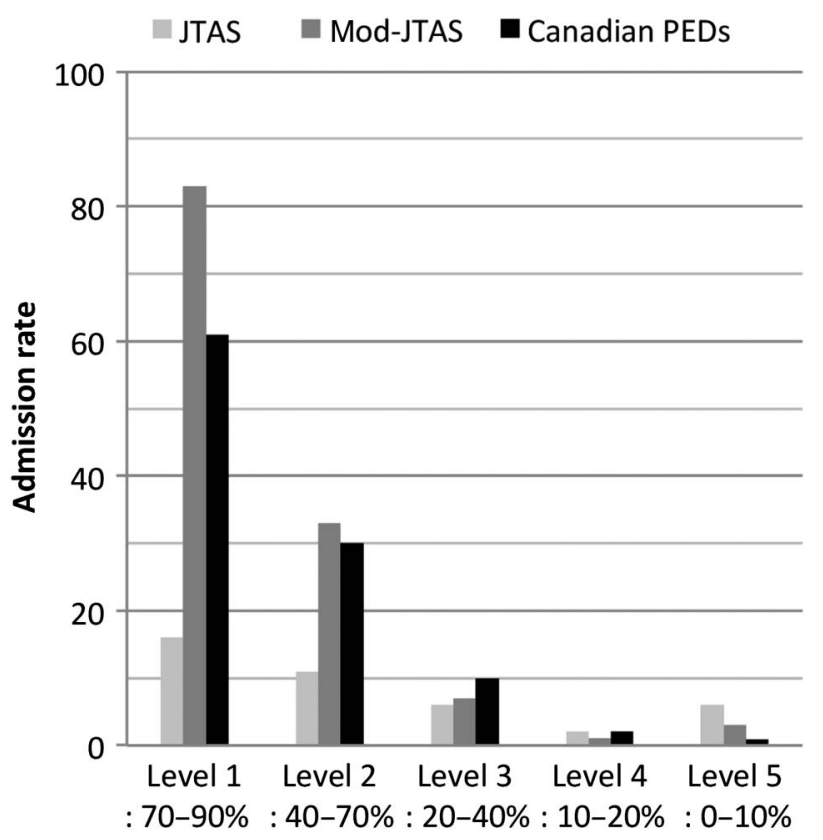

Triage level with anticipated admission rate

Figure 2 Admission rate according to the triage level. Anticipated admission rates defined by Canadian Triage and Acuity Scale guidelines are shown below the triage levels. The black columns represent the admission rate of 12 Canadian PEDs reported by Gravel et al. JTAS, Japanese Triage and Acuity Scale, mod-JTAS, modified JTAS, PED, paediatric emergency department. temperature-pulse centile charts performed poorly among children coming to an ED in the UK, and their utility in distinguishing children with serious infections from those with milder febrile illnesses was poor. ${ }^{11}$

CTAS guidelines discourage use of triage nurses' subjective modification of acuity level, ${ }^{6} 9$ and triaging lower than measured vital signs is allowed 'with caution' (eg, tachycardia in upset child). ${ }^{4}$ The practice of down-triaging must be exercised cautiously, as this is dependent on triage provider's subjective assessment. Lack of experiences or various factors deteriorating nurses' concentration, such as extreme tiredness, work overload or lack of sleep, can cause incorrect decisions. However, our study showed that children in the 'down-triaged' group had similar admission rates as children in 'non-down-triaged' group, which are also comparable with the anticipated rates by CTAS. This reassuring result supports the safe implementation of this practice in our PED to some extent. 'Down-triage' must be reserved for the most experienced triage providers. Further studies are also necessary to establish more detailed 'down-triage' criteria based on objective parameters.

Almost half of patients were 'down-triaged' in our PED during the study period; the vast majority (86\%) were initially assigned higher acuity levels due to tachycardia or tachypnoea. We estimated that one of the reasons for this high proportion of 'down-triaged' patients may be the swift triage process in our PED, due to shortage in space and personnel.

Higher rate of admission in the modified levels 1 and 2 suggests that higher acuity levels were assigned to the patients with truly severe condition, and the rest of the patients who was able to wait longer received lower acuity levels. Therefore, the modification of the initial triage levels allowed better prioritisation of the patients and better allocation of resources in a PED. Appropriate 'down-triage' may assist in further triaging patients to an accurate acuity level, and being seen in a suitable scheduling, based on their perceived condition after examination by an experienced nurse, rather than objective measures of vital signs.

Since triage systems like CTAS and JTAS recommends time to provider, ${ }^{4}$ 'down-triage' of patients results in extending waiting time, with a potential increase in rate of families who LWBS. ${ }^{12}$ However, in our cohort LWBS rate is extremely low $(0.2 \%)$ compared with other reports $(1.6-5.2 \%$ in the US and Canadian paediatric PEDs). ${ }^{10} 13$

CTAS is a consensus-based system developed based on expert opinion. A recent multicentre validation study of CTAS in children by Gravel et al ${ }^{10}$ demonstrated a strong correlation between triage levels and multiple markers of severity. Admission rate has been widely used as a surrogate marker of severity. It is independent of triage and desirable to compare two different triage systems. However, this marker cannot discriminate patients into five urgency categories, and modification of triage categories by scientific approach is yet to be 
validated. ${ }^{14} 15$ Therefore, a validation study of a modified protocol can serve for future improvement of this system. ${ }^{4}$

Our study suggests the possibility of potential down-triaged process in the paediatric ED, for specific groups of children and without increase in admission rate. More research is needed, and if implemented, such down-triaged algorithms need to be refined, carefully monitored and updated on a regular basis, in order to ensure safety of patients and appropriate throughout in the ED.

\section{Limitations}

Our study has several limitations. (1) This was a single-centre retrospective study from Japan, limiting generalisability to other centres. (2) We used admission rate as a surrogate marker of urgency. While this assumption was challenged previously, ${ }^{14-16}$ it is the best available option used by triage validation studies. (3) We could not eliminate the risk of 'down-triaging' truly urgent conditions. Other outcomes such as early PED revisit or subsequent hospitalisation should be considered in the future studies. (4) Some patients who were not triaged, but the rate was $<1 \%$, and unlikely to affect the analysis. (5) 'Down-triage' was determined in almost $8 \%$ of cases based on general impression of the triage nurse, rather than an adjustment for abnormal vital signs. We could not determine a specific protocol used in those cases and variability in down-triage between nurses is a possibility. (6) Finally, the detailed indication of 'down-triage' could not be assessed because of the non-categorised descriptive records. This leaves us an unsolved question of the difference in 'down-triage' per reason for a future study.

\section{CONCLUSION}

In summary, subjective modification of initial triage level apparently results in a more accurate prediction of disposition in our centre in Japan. Revision of triage level seems appropriate if done by experienced personnel. However, further research is needed in order to determine an objective protocol for downtriaging patients to ensure safe practice in a PED.

Acknowledgements The authors acknowledge the contribution of Hideyo Miura, the charge nurse of our PED, for her leadership in the management of 'down-triage' and for supporting the writing of this paper. They thank Boris Kuzeljevic from BC Children's Hospital for supporting the statistical analysis. Finally, they acknowledge Japan-Canada Child Health Exchange Program that helped facilitate the work related to this study.

Contributors TaT, NI, NS and RDG conceived and designed the study. TaT and RDG were involved in analysis and interpretation of data, and drafting the manuscript. NI was involved in acquisition of data, and technical and administrative support. ToT was involved in critical review of the paper. RDG was involved in final proof reading, and study supervision. All authors reviewed and gave final approval of the version to be publisheds.

Competing interests None declared.

Ethics approval Ethical committee of Tokyo Metropolitan Children's Medical Center.

Provenance and peer review Not commissioned; externally peer reviewed.

\section{REFERENCES}

1 Nijman RG, Thompson M, van Veen $\mathrm{M}$, et al. Derivation and validation of age and temperature specific reference values and centile charts to predict lower respiratory tract infection in children with fever: prospective observational study. BMJ 2012;345:e4224.

2 Thompson M, Harnden A, Perera R, et al. Deriving temperature and age appropriate heart rate centiles for children with acute infections. Arch Dis Child 2009;94:361-5.

3 Chang YC, Ng CJ, Wu CT, et al. Effectiveness of a five-level Paediatric Triage System: an analysis of resource utilisation in the emergency department in Taiwan. Emerg Med J 2013;30:735-9.

4 Warren DW, Jarvis A, LeBlanc L, et al. Revisions to the Canadian Triage and Acuity Scale paediatric guidelines (PaedCTAS). CJEM 2008;10:224-43.

5 Gravel J, Manzano S, Arsenault M. Safety of a modification of the triage level for febrile children 6 to 36 months old using the Paediatric Canadian Triage and Acuity Scale. CJEM 2008;10:32-7.

6 Bullard MJ, Chan T, Brayman C, et al. Revisions to the Canadian Emergency Department Triage and Acuity Scale (CTAS) Guidelines. CJEM 2014;16:1-5.

7 Hamamoto J, Yamase H, Yamase Y. Impacts of the introduction of a triage system in Japan: a time series study. Int Emerg Nurs 2014;22:153-8.

8 Fleming S, Thompson M, Stevens $\mathrm{R}$, et al. Normal ranges of heart rate and respiratory rate in children from birth to 18 years of age: a systematic review of observational studies. The Lancet 2011;377:1011-18.

9 Warren D, Jarvis A, LeBlanc L. Canadian Paediatric Triage and Acuity Scale: implementation Guidelines for Emergency Departments. CJEM 2001;3(Suppl 4): S1-27.

10 Gravel J, Fitzpatrick E, Gouin S, et al. Performance of the Canadian Triage and Acuity Scale for children: a multicenter database study. Ann Emerg Med 2013:61:27-32.e3.

11 Brent AJ, Lakhanpaul M, Ninis N, et al. Evaluation of temperature-pulse centile charts in identifying serious bacterial illness: observational cohort study. Arch Dis Child 2011;96:368-73.

12 Goldman RD, Macpherson A, Schuh S, et al. Patients who leave the pediatric emergency department without being seen: a case-control study. CMAJ 2005;172:39-43.

13 Shaw KN, Ruddy RM, Gorelick MH. Pediatric emergency department directors benchmarking survey: fiscal year 2001. Pediatr Emerg Care 2003;19:143-7.

14 Moll HA. Challenges in the validation of triage systems at emergency departments. J Clin Epidemiol 2010;63:384-8.

15 Seiger N, Moll HA. Triage systems: outcome measures to validate. Ann Emerg Med 2013;61:372-3.

16 Pacella CB, Yealy DM. Are we asking the right triage questions? Ann Emerg Med 2013;61:33-4. 